\title{
QUADRATIC FORMS OF DIMENSION 8 WITH TRIVIAL DISCRIMINANT AND CLIFFORD ALGEBRA OF INDEX 4.
}

\author{
ALEXANDRE MASQUELEIN, ANNE QUÉGUINER-MATHIEU, \\ AND JEAN-PIERRE TIGNOL
}

\begin{abstract}
Izhboldin and Karpenko proved in [IK00, Thm 16.10] that any quadratic form of dimension 8 with trivial discriminant and Clifford algebra of index 4 is isometric to the transfer, with respect to some quadratic étale extension, of a quadratic form similar to a two-fold Pfister form. We give a new proof of this result, based on a theorem of decomposability for degree 8 and index 4 algebras with orthogonal involution.
\end{abstract}

Let $W F$ denote the Witt ring of a field $F$ of characteristic different from 2. As explained in [Lam05, X.5 and XII.2], one would like to describe those quadratic forms whose Witt class belongs to the $n$th power $I^{n} F$ of the fundamental ideal $I F$ of $W F$. By the Arason-Pfister Hauptsatz, such a form is hyperbolic if it has dimension $<2^{n}$ and similar to a Pfister form if it has dimension $2^{n}$. More generally, Vishik's Gap Theorem gives the possible dimensions of anisotropic forms in $I^{n} F$.

In addition, one may describe explicitly, for some small values of $n$, low dimensional anisotropic quadratic forms in $I^{n} F$. This is the case, in particular, for $n=2$, that is for even-dimensional quadratic forms with trivial discriminant. In dimension 6, it is well known that such a form is similar to an Albert form, and uniquely determined up to similarity by its Clifford invariant. In dimension 8 , if the index of the Clifford algebra is $\leq 4$, Izhboldin and Karpenko proved in [IK00, Thm 16.10] that it is isometric to the transfer, with respect to some quadratic étale extension, of a quadratic form similar to a two-fold Pfister form.

The purpose of this paper is to give a new proof of Izhboldin and Karpenko's result. Our proof is in the framework of algebras with involution, and does not use Rost's description of 14-dimensional forms in $I^{3} F$ (see [IK00, Rmk 16.11.2]). More precisely, we use triality [KMRT98, (42.3)] to translate the question into a question on algebras of degree 8 and index 4 with orthogonal involution. Our main tool then is a decomposability theorem (Thm. 1.1), proven in $\S 3$. We also use a refinement of a statement of Arason [Ara75, 4.18] describing the even part of the Clifford algebra of a transfer (see Prop. 2.1 below).

\section{Notations and Statement of the theorem}

Throughout the paper, we work over a base field $F$ of characteristic different from 2. We refer the reader to [KMRT98] and [Lam05] for background information on algebras with involution and on quadratic forms. However, we depart from the notation in [Lam05] by using $\left\langle\left\langle a_{1}, \ldots, a_{n}\right\rangle\right\rangle$ to denote the $n$-fold Pfister form $\otimes_{i=1}^{n}\left\langle 1,-a_{i}\right\rangle$. For any quadratic space $(V, \phi)$ over $F$, we let $\operatorname{Ad}_{\phi}$ be the algebra

Date: February 6, 2009

The third author is supported in part by the F.R.S.-FNRS (Belgium). 
with involution $\left(\operatorname{End}_{F}(V), \operatorname{ad}_{\phi}\right)$, where $\operatorname{ad}_{\phi}$ is the adjoint involution with respect to $\phi$, denoted by $\sigma_{\phi}$ in [KMRT98].

For any field extension $L / F$, we denote by $G P_{n}(L)$ the set of quadratic forms that are similar to $n$-fold Pfister forms. This notation extends to the quadratic étale extension $F \times F$ by $G P_{n}(F \times F)=G P_{n}(F) \times G P_{n}(F)$. For any quadratic form $\psi$ over $L$, let $\mathcal{C}(\psi)$ be its full Clifford algebra, with even part $\mathcal{C}_{0}(\psi)$. Both $\mathcal{C}(\psi)$ and $\mathcal{C}_{0}(\psi)$ are endowed with a canonical involution, which is the identity on the underlying vector space, denoted by $\gamma$ (see [KMRT98, p.89]). If $\psi$ has even dimension and trivial discriminant, then its even Clifford algebra splits as a direct product $\mathcal{C}_{+}(\psi) \times \mathcal{C}_{-}(\psi)$, for some isomorphic central simple algebras $\mathcal{C}_{+}(\psi)$ and $\mathcal{C}_{-}(\psi)$ over $F$ (see [Lam05, V, Thm 2.5]). Those algebras are Brauer-equivalent to the full Clifford algebra of $\psi$ and their Brauer class is the Clifford invariant of $\psi$. Assume moreover that $\operatorname{dim}(\psi) \equiv 0 \bmod 4$. As explained in [KMRT98, (8.4)], the involution $\gamma$ then induces an involution on each factor of $\mathcal{C}_{0}(\psi)$, and one may easily check that the isomorphism between the two factors described in the proof of [Lam05, V, Thm 2.5] preserves the involution, so that we actually get a decomposition $\left(\mathcal{C}_{0}(\psi), \gamma\right) \simeq\left(\mathcal{C}_{+}(\psi), \gamma_{+}\right) \times\left(\mathcal{C}_{-}(\psi), \gamma_{-}\right)$, with $\left(\mathcal{C}_{+}(\psi), \gamma_{+}\right) \simeq\left(\mathcal{C}_{-}(\psi), \gamma_{-}\right)$.

Let $L / F$ be a quadratic field extension. For any quadratic form $\psi$ over $L$, we let $\operatorname{tr}_{\star}(\psi)$ be the transfer of $\psi$, associated to the trace map $\operatorname{tr}: L \rightarrow F$, as defined in [Lam05, VII.1.2]. This definition extends to the split étale case $L=F \times F$ and leads to $\operatorname{tr}_{\star}\left(\psi, \psi^{\prime}\right)=\psi+\psi^{\prime}$. On the other hand, for any algebra $A$ over $L$, we let $N_{L / F}(A)$ be its norm, as defined in [KMRT98, §3.B]. Recall that the Brauer class of $N_{L / F}(A)$ is the corestriction of the Brauer class of $A$. Moreover, if $A$ is endowed with an involution of the first kind $\sigma$, then the tensor product $\sigma \otimes \sigma$ restricts to an involution $N_{L / F}(\sigma)$ on $N_{L / F}(A)$. We use the following notation: $N_{L / F}(A, \sigma)=$ $\left(N_{L / F}(A), N_{L / F}(\sigma)\right)$. In the split étale case, we get $N_{F \times F / F}\left((A, \sigma),\left(A^{\prime}, \sigma^{\prime}\right)\right)=$ $(A, \sigma) \otimes\left(A^{\prime}, \sigma^{\prime}\right)($ see $[\mathrm{KMRT} 98, \S 15 . \mathrm{B}])$.

Let $(A, \sigma)$ be a degree 8 algebra with orthogonal involution. We assume that $(A, \sigma)$ is totally decomposable, that is, isomorphic to a tensor product of three quaternion algebras with involution,

$$
(A, \sigma)=\otimes_{i=1}^{3}\left(Q_{i}, \sigma_{i}\right)
$$

If $A$ is split (resp. has index 2), then $(A, \sigma)$ admits a decomposition as above in which each quaternion algebra (resp. each but one) is split (see [Bec08]). Our main result is the following theorem:

Theorem 1.1. Let $(A, \sigma)$ be a degree 8 totally decomposable algebra with orthogonal involution. If the index of $A$ is $\leq 4$, then there exists $\lambda \in F^{\times}$and a biquaternion algebra with orthogonal involution $(D, \theta)$ such that

$$
(A, \sigma) \simeq(D, \theta) \otimes \operatorname{Ad}_{\langle\langle\lambda\rangle\rangle} .
$$

The theorem readily follows from Becher's results mentioned above if $A$ has index 1 or 2 ; it is proven in $\S 3$ for algebras of index 4 . For algebras of index $\leq 2$, we may even assume that $(D, \theta)$ decomposes as a tensor product of two quaternion algebras with involution; this is not the case anymore if $A$ has index 4 , as was shown by Sivatski [Siv05, Prop. 5].

Using triality, we easily deduce the following from Theorem 1.1:

Theorem 1.2 (Izhboldin-Karpenko). Let $\phi$ be an 8-dimensional quadratic form over $F$. The following are equivalent: 
(i) $\phi$ has trivial discriminant and Clifford invariant of index $\leq 4$;

(ii) there exists a quadratic étale extension $L / F$ and a form $\psi \in G P_{2}(L)$ such that $\phi=\operatorname{tr}_{\star}(\psi)$.

If $\phi=\operatorname{tr}_{\star}(\psi)$ for some $\psi \in G P_{2}(L)$, it follows from some direct computation made in $[\mathrm{IK} 00, \S 16]$ that $\phi$ has trivial discriminant and Clifford invariant of index $\leq 4$

Assume conversely that $\phi$ has trivial discriminant. By the Arason-Pfister Hauptsatz, $\phi$ is in $G P_{3}(F)$ if and only if it has trivial Clifford invariant. More generally, it is well-known that $\phi$ decomposes as $\phi=\langle\langle a\rangle\rangle q$ for some $a \in F^{\times}$and some 4dimensional quadratic form $q$ over $F$ if and only if its Clifford invariant has index $\leq 2$ (see for instance [Kne77, Ex 9.12]). Hence, in both cases, $\phi$ decomposes as a sum $\phi=\pi_{1}+\pi_{2}$ of two forms $\pi_{1}, \pi_{2} \in G P_{2}(F)$. This proves that condition (ii) holds with $L=F \times F$.

In section 4 below, we finish this proof by treating the index 4 case. This part of the proof differs from the argument given in [IK00]. In particular, we do not use Rost's description of 14-dimensional forms in $I^{3} F$.

\section{Clifford algebra of the transfer of a Quadratic Form}

Let $L / F$ be a quadratic field extension. By Arason [Ara75, 4.18], for any quadratic form $\psi \in G P_{2}(L)$, the Clifford invariant of the transfer $\operatorname{tr}_{\star}(\psi)$ coincides with the corestriction of the Clifford invariant of $\psi$. In this section, we extend this result, taking into account the algebras with involution rather than just the Brauer classes. More precisely, we prove:

Proposition 2.1. Let $L=F[X] /\left(X^{2}-d\right)$ be a quadratic étale extension of $F$. Consider a quadratic form $\psi$ over $L$ with $\operatorname{dim}(\psi) \equiv 0 \bmod 4$ and $d_{ \pm}(\psi)=1$, so that its even Clifford algebra decomposes as

$$
\left(\mathcal{C}_{0}(\psi), \gamma\right) \simeq\left(\mathcal{C}_{+}(\psi), \gamma_{+}\right) \times\left(\mathcal{C}_{-}(\psi), \gamma_{-}\right), \text {with }\left(\mathcal{C}_{+}(\psi), \gamma_{+}\right) \simeq\left(\mathcal{C}_{-}(\psi), \gamma_{-}\right) .
$$

For any $\lambda \in L^{\times}$represented by $\psi$, the two components of the even Clifford algebra of the transfer of $\psi$ are both isomorphic to

$$
\left(\mathcal{C}_{+}\left(\operatorname{tr}_{\star}(\psi)\right), \gamma_{+}\right) \simeq \operatorname{Ad}_{\left\langle\left\langle-d N_{L / F}(\lambda)\right\rangle\right\rangle} \otimes N_{L / F}\left(\mathcal{C}_{+}(\psi), \gamma_{+}\right) .
$$

Proof. In the split étale case $L=F \times F$, the quadratic form $\psi$ is a couple $\left(\phi, \phi^{\prime}\right)$ of two quadratic forms over $F$ with

$$
\operatorname{dim}(\phi)=\operatorname{dim}\left(\phi^{\prime}\right) \equiv 0 \bmod 4 \quad \text { and } \quad d_{ \pm}(\phi)=d_{ \pm}\left(\phi^{\prime}\right)=1 \in F^{\star} / F^{\star 2} .
$$

Pick $\lambda$ and $\lambda^{\prime}$ in $F$ respectively represented by $\phi$ and $\phi^{\prime}$; the norm $N_{F \times F / F}\left(\lambda, \lambda^{\prime}\right)$ is $\lambda \lambda^{\prime}$. So the following lemma proves the proposition in that case:

Lemma 2.2. Let $\phi$ and $\phi^{\prime}$ be two quadratic forms over $F$ of the same dimension $n \equiv 0 \bmod 4$ and trivial discriminant. For any $\lambda$ and $\lambda^{\prime} \in F^{\times}$, respectively represented by $\phi$ and $\phi^{\prime}$, the components of the even Clifford algebra of the orthogonal sum $\phi+\phi^{\prime}$ are isomorphic to

$$
\left(\mathcal{C}_{+}\left(\phi+\phi^{\prime}\right), \gamma_{+}\right) \simeq \operatorname{Ad}_{\left\langle\left\langle-\lambda \lambda^{\prime}\right\rangle\right\rangle} \otimes\left(\mathcal{C}_{+}(\phi), \gamma_{+}\right) \otimes\left(\mathcal{C}_{+}\left(\phi^{\prime}\right), \gamma_{+}\right) .
$$

Proof of Lemma 2.2. Denote by $V$ and $V^{\prime}$ the underlying quadratic spaces. The natural embeddings $V \hookrightarrow V \oplus V^{\prime}$ and $V^{\prime} \hookrightarrow V \oplus V^{\prime}$ induce $F$-algebra homomorphisms

$$
\mathcal{C}(\phi) \rightarrow \mathcal{C}\left(\phi+\phi^{\prime}\right) \text { and } \mathcal{C}\left(\phi^{\prime}\right) \rightarrow \mathcal{C}\left(\phi+\phi^{\prime}\right) .
$$


One may easily check that the images of the even parts centralize each other, so that we get an $F$-algebra homomorphism

$$
\left(\mathcal{C}_{0}(\phi), \gamma\right) \otimes\left(\mathcal{C}_{0}\left(\phi^{\prime}\right), \gamma\right) \rightarrow\left(\mathcal{C}_{0}\left(\phi+\phi^{\prime}\right), \gamma\right)
$$

Pick orthogonal bases $\left(e_{1}, \ldots, e_{n}\right)$ of $(V, \phi)$ and $\left(e_{1}^{\prime}, \ldots, e_{n}^{\prime}\right)$ of $\left(V^{\prime}, \phi^{\prime}\right)$. The basis of $\mathcal{C}_{0}\left(\phi+\phi^{\prime}\right)$ consisting of products of an even number of vectors of the set $\left\{e_{1}, \ldots, e_{n}, e_{1}^{\prime}, \ldots, e_{n}^{\prime}\right\}$ as described in [Lam05, V, cor 1.9] clearly contains the image of a basis of $\mathcal{C}_{0}(\phi) \otimes \mathcal{C}_{0}\left(\phi^{\prime}\right)$, so that the homomorphism above is injective. In the sequel, we will identify $\mathcal{C}_{0}(\phi)$ and $\mathcal{C}_{0}\left(\phi^{\prime}\right)$ with their images in $\mathcal{C}_{0}\left(\phi+\phi^{\prime}\right)$.

Consider the element $z=e_{1} \ldots e_{n} \in \mathcal{C}_{0}(\phi)$. As explained in [Lam05, V, Thm2.2], for any $v \in V$, one has $v z=-z v \in \mathcal{C}(\phi)$ and $z$ generates the center of $\mathcal{C}_{0}(\phi)$. Since $\phi$ has dimension $0 \bmod 4$ and trivial discriminant, this element $z$ is $\gamma$-symmetric, and multiplying $e_{1}$ by a scalar if necessary, we may assume $z^{2}=1$. The two components of $\mathcal{C}_{0}(\phi)$ are $\mathcal{C}_{+}(\phi)=\mathcal{C}_{0}(\phi) \frac{1+z}{2}$ and $\mathcal{C}_{-}(\phi)=\mathcal{C}_{0}(\phi) \frac{1-z}{2}$. Consider similarly $z^{\prime}=e_{1}^{\prime} \ldots e_{n}^{\prime}$, with $\gamma\left(z^{\prime}\right)=z^{\prime}$ and assume $z^{\prime 2}=1$. The product $z z^{\prime}$ also has square 1 and generates the center of $\mathcal{C}_{0}\left(\phi+\phi^{\prime}\right)$. We denote by $\varepsilon$ the idempotent $\varepsilon=\frac{1+z z^{\prime}}{2}$, so that $\mathcal{C}_{+}\left(\phi+\phi^{\prime}\right)=\mathcal{C}_{0}\left(\phi+\phi^{\prime}\right) \varepsilon$ and $\mathcal{C}_{-}\left(\phi+\phi^{\prime}\right)=\mathcal{C}_{0}\left(\phi+\phi^{\prime}\right)(1-\varepsilon)$.

Let us now fix two vectors $v \in V$ and $v^{\prime} \in V^{\prime}$ such that $\phi(v)=\lambda$ and $\phi^{\prime}\left(v^{\prime}\right)=\lambda^{\prime}$. Since $\frac{1+z}{2} v^{-1}=v^{-1} \frac{1-z}{2}$, we have $v x v^{-1} \in \mathcal{C}_{-}(\phi)$ for any $x \in \mathcal{C}_{+}(\phi)$. Using this identification between the two components, we may diagonally embed $\mathcal{C}_{+}(\phi)$ in $\mathcal{C}_{0}(\phi)$ by considering $x \in \mathcal{C}_{+}(\phi) \mapsto x+v x v^{-1} \in \mathcal{C}_{0}(\phi)$. Similarly, we may embed $\mathcal{C}_{+}\left(\phi^{\prime}\right)$ in $\mathcal{C}_{0}\left(\phi^{\prime}\right)$ by $x^{\prime} \in \mathcal{C}_{+}\left(\phi^{\prime}\right) \mapsto x^{\prime}+v^{\prime} x^{\prime} v^{\prime-1} \in \mathcal{C}_{0}\left(\phi^{\prime}\right)$. Combining those two maps with the morphism

$$
\mathcal{C}_{0}(\phi) \otimes \mathcal{C}_{0}\left(\phi^{\prime}\right) \rightarrow \mathcal{C}_{0}\left(\phi+\phi^{\prime}\right)
$$

and the projection

$$
y \in \mathcal{C}_{0}\left(\phi+\phi^{\prime}\right) \mapsto y \varepsilon \in \mathcal{C}_{+}\left(\phi+\phi^{\prime}\right),
$$

we get an algebra homomorphism

$$
\begin{array}{ccc}
\mathcal{C}_{+}(\phi) \otimes \mathcal{C}_{+}\left(\phi^{\prime}\right) & \rightarrow & \mathcal{C}_{+}\left(\phi+\phi^{\prime}\right) \\
x \otimes x^{\prime} & \mapsto & \left(x+v x v^{-1}\right)\left(x^{\prime}+v^{\prime} x^{\prime} v^{\prime-1}\right) \varepsilon
\end{array}
$$

One may easily check on generators that this map is not trivial; hence it is injective. To conclude the proof, it only remains to identify the centralizer of the image, which by dimension count has degree 2 . It clearly contains $\frac{z+z^{\prime}}{2} \varepsilon$ and $v v^{\prime} \varepsilon$. Moreover, these two elements anticommute, have square $\varepsilon$ and $-\lambda \lambda^{\prime} \varepsilon$, and are respectively symmetric and skew-symmetric under $\gamma$. Hence they generate a split quaternion algebra, with orthogonal involution of discriminant $-\lambda \lambda^{\prime}$, which is isomorphic to $\operatorname{Ad}_{\left\langle\left\langle-\lambda \lambda^{\prime}\right\rangle\right\rangle}$.

This concludes the proof in the split étale case. Until the end of this section, we assume $L$ is a quadratic field extension of $F$, with non-trivial $F$-automorphism denoted by $\iota$. To prove the proposition in this case, we will use the following description of the transfer of a quadratic form and its Clifford algebra.

Let $\psi$ be any quadratic form over $L$, defined on the vector space $V$. We consider its conjugate ${ }^{\iota} V=\left\{{ }^{\iota} v, v \in V\right\}$ with the following operations ${ }^{\iota} v_{1}+{ }^{\iota} v_{2}={ }^{\iota}\left(v_{1}+v_{2}\right)$ and $\lambda \cdot{ }^{\iota} v={ }^{\iota}(\iota(\lambda) \cdot v)$, for any $v_{1}, v_{2}$ and $v$ in $V$ and $\lambda \in L$. Clearly, ${ }^{\iota} \psi\left({ }^{\iota} v\right)=\iota(\psi(v))$ is a quadratic form on ${ }^{\iota} V$. One may easily check from the definition given in [Lam05, VII §1] that the quadratic form $\operatorname{tr}_{\star}(\psi)$ is nothing but the restriction of $\psi+{ }^{\iota} \psi$ to 
the $F$-vector space of fixed points $\left(V \oplus{ }^{\iota} V\right)^{s}$, where $s$ is the switch semi-linear automorphism defined on the direct sum $V \oplus{ }^{\iota} V$ by $s\left(v_{1}+{ }^{\iota} v_{2}\right)=v_{2}+{ }^{\iota} v_{1}$.

Moreover, $s$ induces a semi-linear automorphism of order 2 of the tensor algebra $T\left(V \oplus{ }^{\iota} V\right)$ which preserves the ideal generated by the elements

$$
\left(v_{1}+{ }^{\iota} v_{2}\right) \otimes\left(v_{1}+{ }^{\iota} v_{2}\right)-\left(\psi\left(v_{1}\right)+{ }^{\iota} \psi\left({ }^{\iota} v_{2}\right)\right) .
$$

Hence, we get a semi-linear automorphism $s$ of order 2 on the Clifford algebra $\mathcal{C}\left(\psi+{ }^{\iota} \psi\right)$, which commutes with the canonical involution. The set of fixed points $\left(\mathcal{C}\left(\psi+{ }^{\iota} \psi\right)\right)^{s}$ is an $F$-algebra; the involution $\gamma$ restricts to an $F$-linear involution which we denote by $\gamma_{s}$. We then have:

Lemma 2.3. The natural embedding $\left(V \oplus^{\iota} V\right) \hookrightarrow \mathcal{C}\left(\psi+{ }^{\iota} \psi\right)$, restricted to $\left(V+{ }^{\iota} V\right)^{s}$, induces an isomorphism of graded algebras

$$
\left(\mathcal{C}\left(\operatorname{tr}_{\star}(\psi)\right), \gamma\right) \stackrel{\sim}{\rightarrow}\left(\left(\mathcal{C}\left(\psi+{ }^{\iota} \psi\right)\right)^{s}, \gamma_{s}\right) .
$$

Proof of Lemma 2.3. The natural embedding $\left(V \oplus{ }^{\iota} V\right) \hookrightarrow \mathcal{C}\left(\psi+{ }^{\iota} \psi\right)$ restricts to an injective map $i:\left(V+{ }^{\iota} V\right)^{s} \hookrightarrow \mathcal{C}\left(\psi+{ }^{\iota} \psi\right)^{s}$, which clearly satisfies

$$
i(w)^{2}=\left(\psi+{ }^{\iota} \psi\right)(w) \text { for any } w \in\left(V \oplus{ }^{\iota} V\right)^{s} .
$$

By the universal property of Clifford algebras, it extends to a non-trivial algebra homomorphism $\mathcal{C}\left(\operatorname{tr}_{\star}(\psi)\right) \mapsto \mathcal{C}\left(\psi+{ }^{\iota} \psi\right)^{s}$, which clearly preserves the grading. Since $\mathcal{C}\left(\operatorname{tr}_{\star}(\psi)\right)$ is simple, and both algebras have the same dimension, it is an isomorphism. Clearly, $\gamma$ coincides with $\gamma_{s}$ under this isomorphism.

Hence, we want to describe one component of $\mathcal{C}_{0}\left(\operatorname{tr}_{\star}(\psi)\right) \simeq\left(\mathcal{C}_{0}\left(\psi+{ }^{\iota} \psi\right)\right)^{s}$. We proceed as in the split étale case. Fix an orthogonal basis $e_{1}, \ldots e_{n}$ of $V$ over $L$ such that $\psi\left(e_{n}\right)=\lambda$. The elements ${ }^{\iota} e_{1}, \ldots,{ }^{\iota} e_{n}$ are an orthogonal basis of ${ }^{\iota} V$ and ${ }^{\iota} \psi\left({ }^{\iota} e_{n}\right)=\iota(\lambda)$. We may moreover assume that $z=e_{1} \ldots e_{n}$ and ${ }^{\iota} z={ }^{\iota} e_{1} \ldots{ }^{\iota} e_{n}$ have square 1 . Since the idempotent $\varepsilon=\frac{1+z^{\iota} z}{2} \in C_{0}\left(\psi+{ }^{\iota} \psi\right)$ satisfies $s(\varepsilon)=\varepsilon$, the semilinear automorphism $s$ preserves each factor $\mathcal{C}_{+}\left(\psi+{ }^{\iota} \psi\right)$ and $\mathcal{C}_{-}\left(\psi+{ }^{\iota} \psi\right)$. Hence, the components of $\mathcal{C}_{0}\left(\operatorname{tr}_{\star}(\psi)\right)$ are

$$
\mathcal{C}_{0}\left(\operatorname{tr}_{\star}(\psi)\right)=\left(\mathcal{C}_{+}\left(\psi+{ }^{\iota} \psi\right)\right)^{s} \times\left(\mathcal{C}_{-}\left(\psi+{ }^{\iota} \psi\right)\right)^{s} .
$$

Moreover, by Lemma 2.2, we have

$$
\mathcal{C}_{+}\left(\psi+{ }^{\iota} \psi\right) \simeq \operatorname{Ad}_{\langle\langle-\lambda \iota(\lambda)\rangle\rangle} \otimes\left(\mathcal{C}_{+}(\psi), \gamma\right) \otimes\left(\mathcal{C}_{+}\left({ }^{\iota} \psi\right), \gamma\right),
$$

and it remains to understand the action of the switch automorphism on this tensor product. First, one may identify $\mathcal{C}_{+}\left({ }^{\iota} \psi\right)$ with the algebra ${ }^{\iota} \mathcal{C}_{+}(\psi)$ defined by

$$
{ }^{\iota} \mathcal{C}_{+}(\psi)=\left\{{ }^{\iota} x, x \in \mathcal{C}_{+}(\psi)\right\}
$$

with the operations

$$
{ }^{\iota} x+{ }^{\iota} y={ }^{\iota}(x+y), \quad{ }^{\iota} x^{\iota} y={ }^{\iota}(x y) \text { and }{ }^{\iota}(\lambda x)=\iota(\lambda)^{\iota} x,
$$

for all $x, y \in \mathcal{C}_{+}(\psi)$ and $\lambda \in L$. Clearly, the switch automorphism acts on the tensor product

by

$$
\mathcal{C}_{+}(\psi) \otimes \mathcal{C}_{+}\left({ }^{\iota} \psi\right) \simeq \mathcal{C}_{+}(\psi) \otimes{ }^{\iota} \mathcal{C}_{+}(\psi)
$$

$$
s\left(x \otimes{ }^{\iota} y\right)=y \otimes{ }^{\iota} x,
$$

and by definition of the corestriction (see [KMRT98, 3.B]), the F-subalgebra of fixed points is

$$
\left(\left(\mathcal{C}_{+}(\psi), \gamma\right) \otimes\left({ }^{\iota} \mathcal{C}_{+}(\psi), \gamma\right)\right)^{s}=N_{L / F}\left(\mathcal{C}_{+}(\psi), \gamma\right)
$$


It remains to understand the action of the switch on the centralizer, which is the split quaternion algebra over $L$ generated by $x=\frac{z+^{\iota} z}{2} \varepsilon$ and $y=e_{n}{ }^{\iota} e_{n} \varepsilon$. The element $x$ clearly is $s$-symmetric, while $y$ satisfies $s(y)=-y$. Let $\delta$ be a generator of the quadratic extension $L / F$, so that $\iota(\delta)=-\delta$ and $\delta^{2}=d$. Since the switch map $s$ is $L / F$ semi-linear, we may replace $y$ by $\delta y$ which now satisfies $s(\delta y)=\delta y$. Hence, the set of fixed points under $s$ is the split quaternion algebra over $F$ generated by $x$ and $\delta y$. Since $(\delta y)^{2}=-d N_{L / F}(\lambda)$, it is isomorphic to $\operatorname{Ad}_{\left\langle\left\langle-d N_{L / F}(\lambda)\right\rangle\right\rangle}$.

\section{Proof of the DeComposability THeOREM}

In this section, we finish the proof of Theorem 1.1. Let $(A, \sigma)=\otimes_{i=1}^{3}\left(Q_{i}, \sigma_{i}\right)$ be a product of three quaternion algebras with orthogonal involution. We assume that $A$ has index 4, so that it is Brauer-equivalent to a biquaternion division algebra $D$. We have to prove that $(A, \sigma)$ is isomorphic to $(D, \theta) \otimes \operatorname{Ad}_{\langle\langle\lambda\rangle\rangle}$ for a well chosen involution $\theta$ on $D$ and some $\lambda \in F^{\times}$.

The algebra $D$ is endowed with an orthogonal involution $\tau$, and we may represent

$$
(A, \sigma)=\left(\operatorname{End}_{D}(M), \operatorname{ad}_{h}\right)
$$

for some 2-dimensional hermitian module $(M, h)$ over $(D, \tau)$. Let us consider a diagonalisation $\left\langle a_{1}, a_{2}\right\rangle$ of $h$, and define

$$
\theta=\operatorname{Int}\left(a_{1}^{-1}\right) \circ \tau \text {. }
$$

With respect to this new involution, we get another representation

$$
(A, \sigma)=\left(\operatorname{End}_{D}(M), \operatorname{ad}_{h^{\prime}}\right)
$$

where $h^{\prime}$ is a hermitian form over $(D, \theta)$ which diagonalises as $h^{\prime}=\langle 1,-a\rangle$ for some $\theta$-symmetric element $a \in D^{\times}$. The theorem now follows from the following lemma:

Lemma 3.1. The involutions $\theta$ and $\theta^{\prime}=\operatorname{Int}\left(a^{-1}\right) \circ \theta$ of the biquaternion algebra $D$ are conjugate.

Indeed, assume there exists $u \in A^{\times}$such that $\theta=\operatorname{Int}(u) \circ \theta^{\prime} \circ \operatorname{Int}\left(u^{-1}\right)$. We then have $\theta=\operatorname{Int}\left(u a^{-1}\right) \circ \theta \circ \operatorname{Int}\left(u^{-1}\right)=\theta \circ \operatorname{Int}\left(\theta(u)^{-1} a u^{-1}\right)$. Hence, there exists $\lambda \in F^{\times}$such that $\theta(u)^{-1} a u^{-1}=\lambda$, that is $a=\lambda \theta(u) u$. This implies that the hermitian form $h^{\prime}=\langle 1,-a\rangle$ over $(D, \theta)$ is isometric to $\langle 1,-\lambda\rangle$. Since $\lambda \in F^{\times}$, we get $(A, \sigma)=\left(\operatorname{End}_{D}(M), \operatorname{ad}_{\langle 1,-\lambda\rangle}\right)=(D, \theta) \otimes \operatorname{Ad}_{\langle\langle\lambda\rangle\rangle}$, and it only remains to prove the lemma.

Proof of Lemma 3.1. We want to compare the orthogonal involutions $\theta$ and $\theta^{\prime}$ of the biquaternion algebra $D$. By [LT99, Prop. 2], they are conjugate if and only if their Clifford algebras $\mathcal{C}$ and $\mathcal{C}^{\prime}$ are isomorphic as $F$-algebras. This can be proven as follows.

Since $(A, \sigma)$ is a product of three quaternion algebras with involution, we know from [KMRT98, (42.11)] that the discriminant of $\sigma$ is 1 and its Clifford algebra has one split component.

On the other hand, the representation $(A, \sigma)=\left(\operatorname{End}_{D}(M), \operatorname{ad}_{\langle 1,-a\rangle}\right)$ tells us that $(A, \sigma)$ is an orthogonal sum, as in [Dej95], of $(D, \theta)$ and $\left(D, \theta^{\prime}\right)$. Hence its invariants can be computed in terms of those of $(D, \theta)$ and $\left(D, \theta^{\prime}\right)$. By [Dej95, Prop. 2.3.3], the discriminant of $\sigma$ is the product of the discriminants of $\theta$ and $\theta^{\prime}$. So $\theta$ and $\theta^{\prime}$ have the same discriminant, and we may identify the centers $Z$ and $Z^{\prime}$ of their Clifford algebras in two different ways. We are in the situation described in [LT99, p. 265], where the Clifford algebra of such an orthogonal sum is computed. In 
particular, since one component of the Clifford algebra of $(A, \sigma)$ is split, it follows from [LT99, Lem 1] that

$$
\mathcal{C} \simeq \mathcal{C}^{\prime} \quad \text { or } \quad \mathcal{C} \simeq{ }^{\iota} \mathcal{C}^{\prime},
$$

depending on the chosen identification between $Z$ and $Z^{\prime}$. In both cases, $\mathcal{C}$ and $\mathcal{C}^{\prime}$ are isomorphic as $F$-algebras, and this concludes the proof.

\section{A NeW PRoOf of IzhBoldin And Karpenko's theOREM}

Let $\phi$ be an 8-dimensional quadratic form over $F$ with trivial discriminant and Clifford invariant of index 4 . We denote by $(A, \sigma)$ one component of its even Clifford algebra, so that

$$
\left(\mathcal{C}_{0}(\phi), \gamma\right) \simeq(A, \sigma) \times(A, \sigma)
$$

where $A$ is an index 4 central simple algebra over $F$, with orthogonal involution $\sigma$.

By triality [KMRT98, (42.3)], the involution $\sigma$ has trivial discriminant and its Clifford algebra is

$$
\mathcal{C}(A, \sigma)=\operatorname{Ad}_{\phi} \times(A, \sigma) .
$$

In particular, it has a split component, so that the algebra with involution $(A, \sigma)$ is isomorphic to a tensor product of three quaternion algebras with involution (see [KMRT98, (42.11)]). Hence we can apply our decomposability theorem 1.1, and write $(A, \sigma)=(D, \theta) \otimes \operatorname{Ad}_{\langle\langle\lambda\rangle\rangle}$ for some biquaternion division algebra with orthogonal involution $(D, \theta)$ and some $\lambda \in F^{\times}$.

Let us denote by $d$ the discriminant of $\theta$, and let $L=F[X] /\left(X^{2}-d\right)$ be the corresponding quadratic étale extension. Consider the image $\delta$ of $X$ in $L$. By Tao's computation of the Clifford algebra of a tensor product [Tao95, Thm. 4.12], the components of $\mathcal{C}(A, \sigma)$ are Brauer-equivalent to the quaternion algebra $(d, \lambda)$ over $F$ and the tensor product $(d, \lambda) \otimes A$. Since $A$ has index 4, the split component has to be $(d, \lambda)$, so that $\lambda$ is a norm of $L / F$, say $\lambda=N_{L / F}(\mu)$.

Consider now the Clifford algebra of $(D, \theta)$. It is a quaternion algebra $Q$ over $L$, endowed with its canonical (symplectic) involution $\gamma$. Denote by $n_{Q}$ the norm form of $Q$, that is $n_{Q}=\langle\langle\alpha, \beta\rangle\rangle$ if $Q=(\alpha, \beta)_{L}$. It is a 2 -fold Pfister form and for any $\ell \in L^{\star},\left(\mathcal{C}_{+}\left(\langle\ell\rangle n_{Q}\right), \gamma_{+}\right) \simeq(Q, \gamma)$. Moreover, by the equivalence of categories $A_{1}^{2} \equiv D_{2}$ described in [KMRT98, (15.7)], the algebra with involution $(D, \theta)$ is canonically isomorphic to $N_{L / F}(Q, \gamma)$.

Hence we get that $(A, \sigma)=N_{L / F}(Q, \gamma) \otimes \operatorname{Ad}_{\left\langle\left\langle-d N_{L / F}(\delta \mu)\right\rangle\right.}$. By Proposition 2.1, this implies that

$$
(A, \sigma) \times(A, \sigma) \simeq\left(\mathcal{C}_{0}\left(\operatorname{tr}_{\star}(\psi)\right), \gamma\right),
$$

where $\psi=\langle\delta \mu\rangle n_{Q}$. Applying again triality [KMRT98, (42.3)], we get that the split component $\operatorname{Ad}_{\phi}$ of the Clifford algebra of $(A, \sigma)$ also is isomorphic to $\operatorname{Ad}_{t_{*}(\psi)}$, so that the quadratic forms $\phi$ and $\operatorname{tr}_{\star}(\psi)$ are similar. This concludes the proof since $\psi$ belongs to $G P_{2}(L)$.

Remark. Let $\phi$ and $(A, \sigma)$ be as above, and let $L=F[X] /\left(X^{2}-d\right)$ be a fixed quadratic étale extension of $F$. It follows from the proof that the quadratic form $\phi$ is isometric to the transfer of a form $\psi \in G P_{2}(L)$ if and only if $(A, \sigma)$ admits a decomposition $(A, \sigma)=\operatorname{Ad}_{\langle\langle\lambda\rangle\rangle} \otimes(D, \theta)$, with $d_{ \pm}(\theta)=d$. In particular, the quadratic form $\phi$ is a sum of two forms similar to 2 -fold Pfister forms exactly when the algebra with involution $(A, \sigma)$ admits a decomposition as $(D, \theta) \otimes \operatorname{Ad}_{\langle\langle\lambda\rangle\rangle}$ with $\theta$ of 
discriminant 1 , that is when it decomposes as a tensor product of three quaternion algebras with involution, with one split factor.

Such a decomposition does not always exist, as was shown by Sivatski [Siv05, Prop 5]. This reflects the fact that 8-dimensional quadratic forms $\phi$ with trivial discriminant and Clifford algebra of index $\leq 4$ do not always decompose as a sum of two forms similar to two-fold Pfister forms (see [IK00, §16] and [HT98] for explicit examples).

\section{REFERENCES}

[Ara75] J. K. ARASON - "Cohomologische Invarianten quadratischer Formen", J. Alg. 36 (1975), p. 448-491.

[Bec08] K. J. BECHER - "A proof of the Pfister factor conjecture", Invent. Math. 173 (2008), no. 1, p. $1-6$.

[Dej95] I. DEJAIFFE - "Somme orthogonale d'algèbres à involution et algèbre de Clifford", Comm. Algebra 26(5) (1995), p. 1589-1612.

[HT98] D. W. Hoffmann et J.-P. Tignol - "On 14-dimensional quadratic forms in $I^{3}, 8$ dimensional forms in $I^{2}$, and the common value property", Doc. Math. 3 (1998), p. 189-214 (electronic).

[IK00] O. T. Izhboldin et N. A. KARPENKo - "Some new examples in the theory of quadratic forms", Math. Z. 234 (2000), no. 4, p. 647-695.

[KMRT98] M.-A. Knus, S. Merkurjev, M. Rost et J.-P. Tignol - The book of involutions, Colloquium Publ., vol. 44, Amer. Math. Soc., Providence, RI, 1998.

[Kne77] M. Knebusch - "Generic splitting of quadratic forms. II", Proc. London Math. Soc. (3) 34 (1977), no. 1, p. 1-31.

[Lam05] T.-Y. LAM - Introduction to quadratic forms over fields, Grad. Studies in Math., vol. 67, Amer. Math. Soc., 2005.

[LT99] D. W. LEWIS et J.-P. TignOL - "Classification theorems for central simple algebras with involution", Manuscripta Math. 100 (1999), no. 3, p. 259-276, With an appendix by R. Parimala.

[Siv05] A. S. SivATSKI - "Applications of Clifford algebras to involutions and quadratic forms", Comm. Algebra 33 (2005), no. 3, p. 937-951.

[Tao95] D. TAO - "The generalized even Clifford algebra", J. Algebra 172 (1995), no. 1, p. $184-204$.

Département de Mathématiques, Université Catholique de Louvain, Chemin du CyClotron, 2, B1348 Louvain-la-Neuve, Belgique

E-mail address: Alexandre.Masquelein@uclouvain.be

Université Paris 13 (LAGA), CNRS (UMR 7539), Université Paris 12 (IUFM), 93430 Villetaneuse, France

E-mail address: queguin@math.univ-paris13.fr

$U R L:$ http://www-math.univ-paris13.fr/ queguin/

Département de Mathématiques, Université Catholique de Louvain, Chemin du CyClotron, 2, B1348 Louvain-la-Neuve, Belgique

E-mail address: jean-pierre.tignol@uclouvain.be

$U R L:$ http://wwww .math.ucl.ac.be/membres/tignol 